Research article

\title{
Role of dicot angiosperms in the livelihood of Mishing community in Sonitpur district, Assam, India
}

\author{
Jintu Sarma and Ashalata Devi* \\ Ecology and Biodiversity Lab., Department of Environmental Science, Tezpur University, \\ Napaam, Tezpur-784028, Assam, India \\ *Corresponding Author: ashalatadevi12@gmail.com \\ [Accepted: 12 December 2016]

\begin{abstract}
Assam, a state in Northeast India holds and supports a high percentage of tribal population, highly distinguished in terms of ethno-lingual physiognomies as well as livelihood status in their habitats. The Mishing people or Mising, also called Miri, is a major ethnic tribal community inhabiting in the Sonitpur district of Assam and the second largest tribal group in Northeast India. The livelihoods of Mishing community are closely associated with several plant species. The present study was carried out in the Sonitpur district of Assam, India to trace out the role of dicot angiosperm in the livelihood pattern of Mishing community living in the area. Through a series of extensive survey a total number of ethnobotanically important dicot plant species under 64 genera and 45 families were recorded. 48 dicot plant species were exclusively used for medicinal purposes and 25 dicot plant species were found to be marketed for different purposes as NTFPs. The uses and marketing of different Non Timber Forest Products (NTFPs) were also recorded to determine their economic reliance. While, 11 species of dicot angiosperm were recorded that are used for the preparation of Rogjin Apong, an ethnic alcoholic rice bear.
\end{abstract}

Keywords: Dicot Angiosperms - Mishing Community - Livelihood - Sonitpur - Assam.

[Cite as: Sarma J \& Devi A (2016) Role of dicot angiosperms in the livelihood of Mishing community in Sonitpur district, Assam, India. Tropical Plant Research 3(3): 662-672]

\section{INTRODUCTION}

India has about 10.05 crore tribal population, consisting $8.60 \%$ of country's total population (Census 2011). The tribal population in India contains approximately 250 groups, speaking about 105 languages and 225 subsidiary languages. In the context of socio-economic development, the tribals in India vary from primitive life style to modern way of living. The primitive tribal economy is intimately connected with forests and its resources. Tribal's and their symbiotic relationship with forest and their surrounding environment has been seen through nature worship since ancient times. Nature gives many things such as food, medicine, raw materials, shelter, fertilizer, fuel, timber etc. Thus tribal people are dependent on these natural resources for their livelihood and sustenance. Many workers reported that tribal peoples are highly dependent on NTFPs for the source of income and livelihood (Rao 1987, Gauraha 1992, Chopra 1993, Mallik 2000). About 60 per cent of NTFPs is consumed by about 7 crore tribals or ethnic communities in the country and reported to contribute about 10 to $40 \%$ of their household earnings (Shiva 1993). About $70 \%$ of Indian population dwells in rural areas and many of them rely on various non-timber forests products for their sustenance (Datta et al. 2014). Medicinal plants have a long-standing history among indigenous communities and are an integral part for treating various diseases, particularly to curb/cure daily ailments and this practice of traditional medicine is based on experience of hundreds of years of belief and observations. Indigenous healing practices have been culturally accepted during all phases of human culture and environmental evolution. Traditional medicine is widely used since prehistoric period (Singh \& Lahiri 2010) and accounts for about $40 \%$ of all health care delivered (WHO 2005). It has also been estimated that about $85 \%$ of worldwide traditional medicines are derived from plants (Fransworth 1988). Majority of different tribal community of Indian population depend directly or indirectly on about 7500 different medicinal plants for the treatments of their various health ailments. 
India is one of the great treasures of ethnobotanical wealth having ultimate multiplicity of ethnic community and highly engrossed biological (Kala 2005) resources. In developing countries, the use of herbal treatment is enormously increasing day by day and many modern researchers are trying to intricate and explore the huge potential of ethnobotanical knowledge of medicinal plants for treatment of various diseases (Kala 2005, Dutta \& Dutta 2005, Jain et al. 2010, Jeyaprakash et al. 2011, Mehra et al. 2014, Bajpai et al. 2016, Ngbolua et al. 2016). However, the ethnomedicinal plants in their natural habitat are under threat due to deforestation, overgrazing and their reckless harvesting and utilization. Several workers carried out, ethnobotanical studies with reference to the uses of medicinal plants by different tribal communities from various places of Assam (Jain \& Borthakur 1980, Jain 1987, Jain 1989, Das 2008, Saikia et al. 2010, Rout 2012, Teron \& Borthakur 2014). Among other tribes Mishing is a major tribal community of Sonitpur district whose livelihood is highly associated with the plants for medicine, food and for daily basic needs. The main source of livelihood for the Mishing community is agriculture. An attempt has been made to examine the dependency of Mishing community on several plants for medicinal uses values and NTFPs for marketing as one of their livelihood options in Sonitpur district of Assam, India.

\section{MATERIALS AND METHODS}

Study area

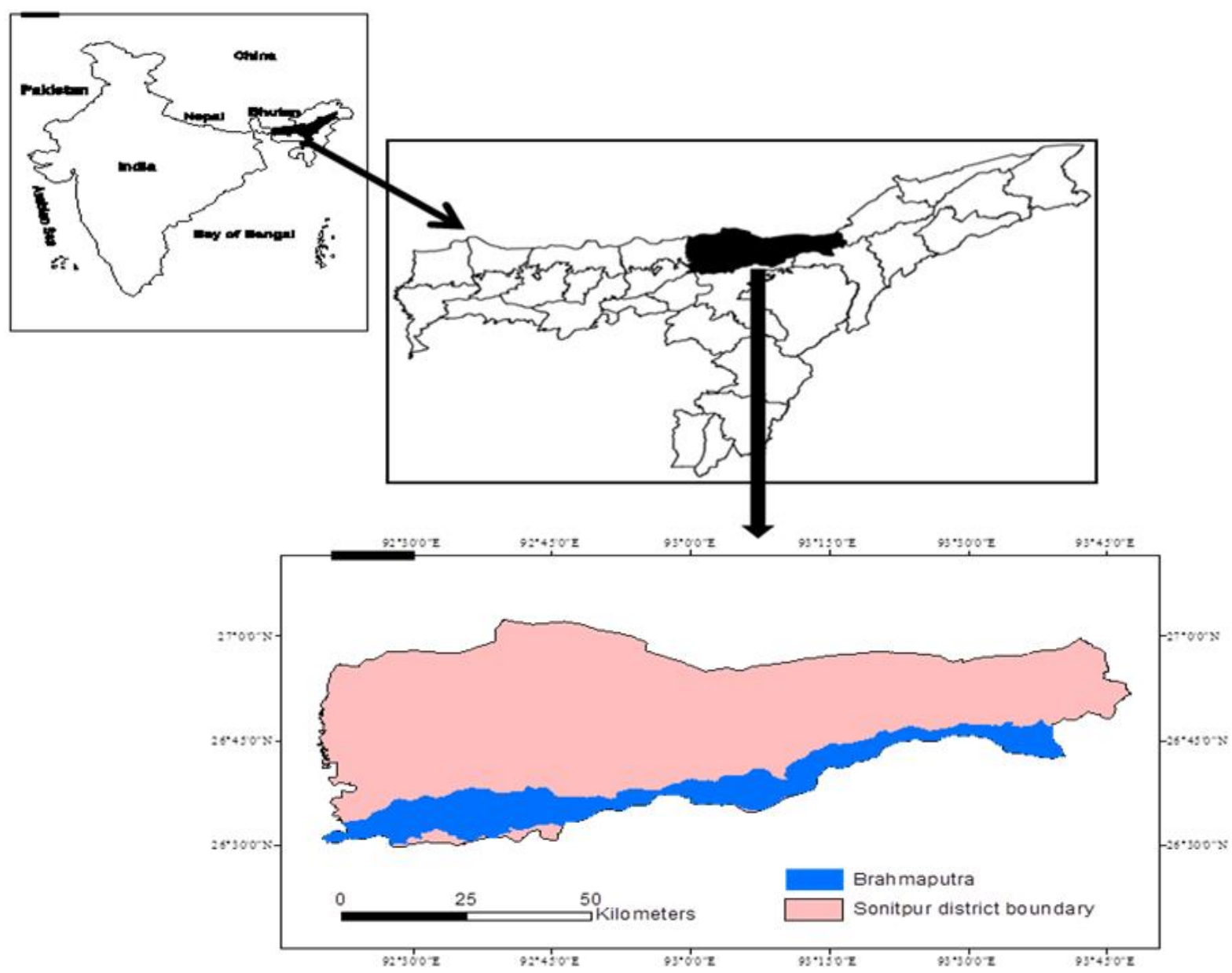

Figure 1. Map of study area, Sonitpur district of Assam, India.

Sonitpur is the second largest district of state Assam after Karbi Anglong district, spread over an area of $5324 \mathrm{~km}^{2}$ on the north bank of Brahmaputra river with a population about 1.926 million (Census 2011). The Sonitpur district lies between $26^{\circ} 40^{\prime} 25.9860^{\prime \prime} \mathrm{N}$ and 92 ${ }^{\circ}$ 51' 27.7560" E and is bounded by Arunachal Pradesh in north, River Brahmaputra in south; Lakhimpur in east and Darrang in the west (Fig. 1). There are five major Rivers of Sonitpur namely, Brahmaputra, Jiabharali, Gabharu, Borgang and Buroi. The total forest area of 
Sonitpur is $1420 \mathrm{~km}^{2}$. The major forest area of district Sonitpur comprises Nameri National Park (NP); Burhachapori and Sonai Rupai Wildlife Sanctuaries (WLS) and 11 Reserve Forests (RF) distributed in two broad divisions Sonitpur East and West. As per census 2011 data the tribal population of Sonitpur is about 2,32,000 which comprises of different tribal groups, among them Mishing, Bodo, Rabhas, Mechs, Nyishis, Garos, Adis, Munda, Apatanis, Lamas etc. are some important tribal communities.

\section{Methodology}

An extensive field survey was carried out in order to document ethnobotanical plant species associated with Mishing community in seventeen (17) different villages during 2013 - 2015. A series of informal meeting was conducted with the village heads of each Mishing village and villagers with the age group of 15-85. A standard semi-structured questioner was used for the proposed survey which was prepared following the guideline of Convention on Biological Diversity (CBD). Vernacular names (Mishing) of plants were recorded while for medicinal plants part used, mode of administration, dose recommended for human care, nature and name of diseases were properly recorded through personal interaction with medicine men. For the record of NTFPs available in markets several small local markets (Bhalukpong, Khonamukh, Chariduar, near Gohpur, etc.) were also visited at frequent interval. Whole plants and different plant parts sold in the local markets were recorded and prices of each item were quantified. Herbarium were prepared following Jain \& Rao (1977) for the recorded plant species and were identified checking the specimens at the herbarium of Gauhati University, Assam, Botanical Survey of India, Shillong and consulting relevant literature such as 'Flora of British India' (Hooker 1875-1894), 'Flora of Assam' (Kanjilal et al. 1934-1940), etc. and also consulted with some highly experienced taxonomists like Dr. Gajen Chandra Sarma, Gauhati University, Assam, India. The analysed herbariums were submitted to the Tezpur University Herbarium (TUH) at Ecology and Biodiversity laboratory for preservation.

\section{RESULTS}

The results of the present study highlight the ethno-medicine and NTFPs used by Mishing community of Sonitpur districts. The survey was carried out in 17 villages viz. Dharikati, Khonamukh, Kathani, Rangajan, Rongajan miri, Baligaon, Sotaimiri, Toupamiri, Bamunipam, Bordikorai, Sikomgaon, Silenighat, Morikhuti, Bokagaon, Kekokoli, Tinighoria and Gudamghat. A total of 194 villagers (149 male and 45 female) were interviewed. 55 respondents in the interview process belonged to age group of $32-45$ years; 70 individuals in the range of 46-55 years; 33 individuals of 56-65 years; 24 individuals in 66-75 years and only 12 individuals in between 76-85 years. During this study a total number of 74 ethnobotanically important dicot plant species under 64 genera and 45 families has been recorded. Among this, 48 species were medicinal plant belonging to 46 genera under 34 families that were exclusively used for the ethno-medicinal purposes by the Mishing community (Table 1). Trees contributed highest (42\%) number having 20 species belonging to 18 genera and 15 families followed by shrubs ( $31 \%$; 15 species under 15 genera and 11 families), herbs (17\%; 8 species, 8 genera and 8 families) and climber (10\%) with 5 species, 5 genera and 5 families. Among the families Acanthaceae shows highest species diversity ( 4 genera and 4 species) followed by Rutaceae ( 3 genera and 3 species), Verbenaceae and Moraceae ( 2 genera and 3 species each) and Combretaceae has 3 species under 1 genera. While Euphorbiaceae, Lythraceae, Meliaceae, Solanaceae, Lamiaceae, Rubiaceae holds 2 genera and 2 species each. The other families comprise 1 genera and single species (Fig. 2). The different plant parts used for the different treatment of human diseases are also recorded during the study. Among the plant part, leaves (50\%) is extensively used as medicines followed by fruit (17\%), whole plant, root and stem (6\%), bark (7\%), gum/resin $(4 \%)$, flower and seed consists (2\%), as given in figure 3. During the study a detailed note on the species used for different diseases were also estimated. As per record, $19 \%$ of species were utilised for stomach problems; $13 \%$ utilised for dysentery; $10 \%$ were used for skin problems; $8 \%$ for hair therapy, worm and cough; $4 \%$ were used in liver disorder, gynaecological, urinary problems, infections and as health tonic each; and $2 \%$ of recorded species were used for diabetes, dog-bite, pains, teeth problems, abortion and fractures (Fig. 4). During the interview it has also been observed that the majority of the medicine prepared by herbal medicine-men was administrated internally contributing $81 \%$ and some $19 \%$ medicine were administrated externally. Another part of this study was to trace out different NTFPs specially dicots from Sonitpur district used by Mishing tribe. After a multi phased field observation a total of 23 species belonging to 17 genera under 15 families were www.tropicalplantresearch.com 


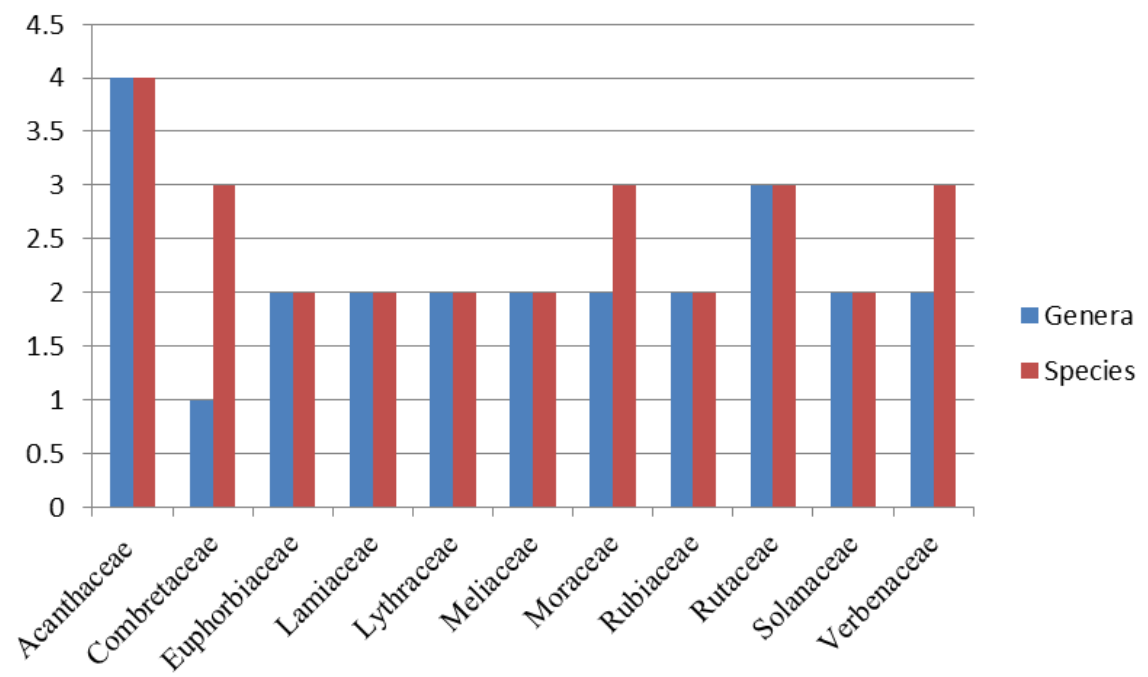

Figure 2. Analysis of dominant families (Dicots) for the recorded plant species.

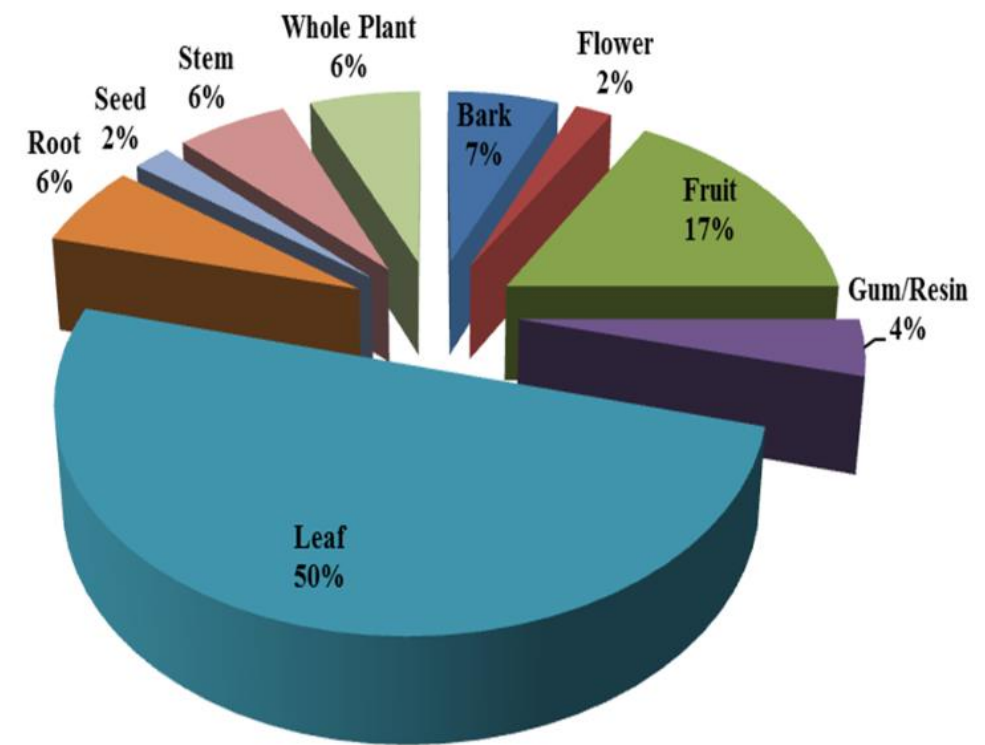

Figure 3. Percentage contribution of different plant parts used in preparation of medicine.

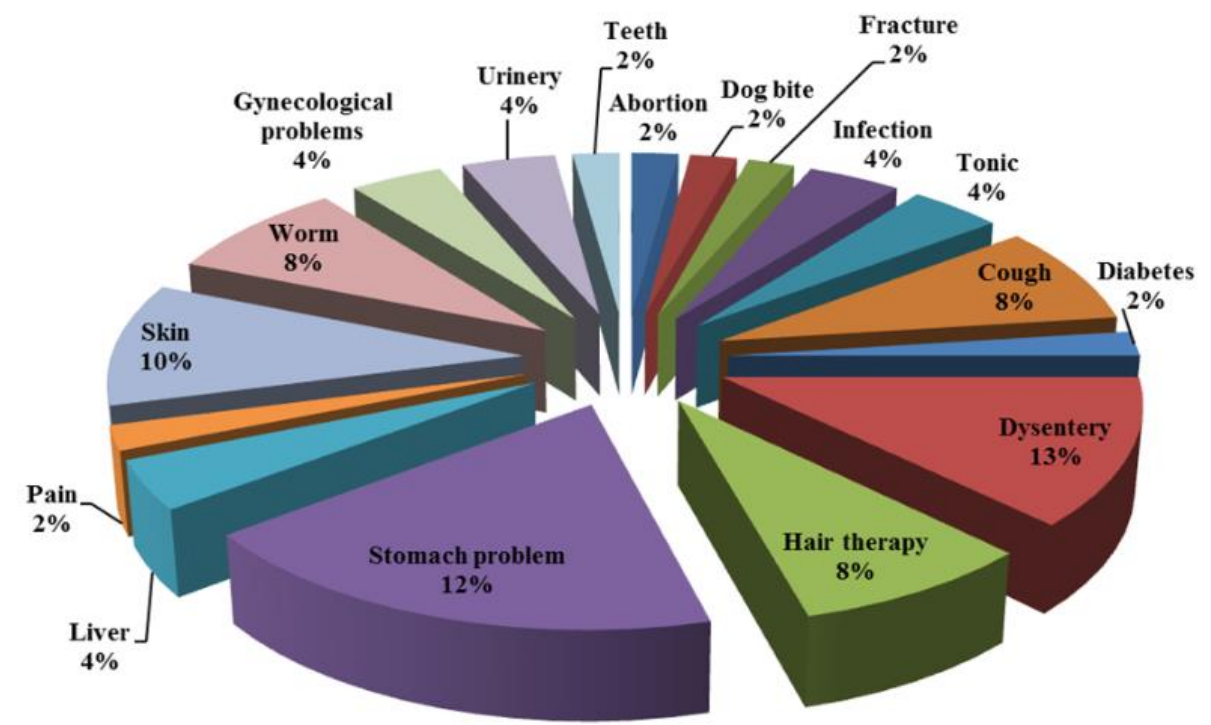

Figure 4. Contribution of medicinal plants used for treatments of certain human health ailments. 
Table 1. Details of the recorded ethno-medicinal plant species used by Mishing tribe. (Note: T- Tree, CL- Climber, SH- Shrub, H- Herb)

\begin{tabular}{|c|c|c|c|c|c|c|c|}
\hline $\begin{array}{l}\text { Sl. } \\
\text { No. }\end{array}$ & $\begin{array}{l}\text { Botanical name } \\
\text { of the plants }\end{array}$ & Family & $\begin{array}{l}\text { Vern name } \\
\text { (Mishing) }\end{array}$ & Uses & $\begin{array}{c}\text { Life } \\
\text { form }\end{array}$ & Part used & Dose/ administration \\
\hline 1. & Acacia nilotica (L.) Delile & Mimosaceae & Babul & $\begin{array}{l}\text { Dry cough, } \\
\text { kidney trouble }\end{array}$ & $\mathrm{T}$ & Leaf, bark & I: decoction of leaf and bark used after meal. \\
\hline 2. & Adhatoda vasica Nees & Acanthaceae & Bahek phul & Cough etc. & $\mathrm{H}$ & Leaf & I: decoction of leaf used after meal. \\
\hline 3. & Aegle marmelos (L.) Corréa & Rutaceae & Bel & Small pox & $\mathrm{T}$ & Leaf & E: pest of young leaf used in the small pox. \\
\hline 4. & Ananas comosus (L.) Merr. & Bromeliaceae & Matikothal & $\begin{array}{l}\text { Vomiting } \\
\text { /indigestion }\end{array}$ & $\mathrm{SH}$ & Leaf & $\begin{array}{l}\text { I: } 2 / 3 \text { young leaf taken and grind then the juice is } \\
\text { directly taken with a small salt. }\end{array}$ \\
\hline 5. & $\begin{array}{l}\text { Andrographis paniculata } \\
\text { (Burm. f.) Nees }\end{array}$ & Acanthaceae & Kalmegh & Liver tonic & SH & Leaf & $\begin{array}{l}\text { I: young leaf smashed and the distilled juice is } \\
\text { taken daily for liver problem. }\end{array}$ \\
\hline 6. & Asparagus racemosus Willd. & Asperagaceae & Satmul & $\begin{array}{l}\text { Upset stomach } \\
\text { (dyspepsia), } \\
\text { constipation }\end{array}$ & CL & Root & $\begin{array}{l}\text { I: root boiled with water or dried first and then } \\
\text { powder boiled in water and taken for stomach } \\
\text { problems. }\end{array}$ \\
\hline 7. & Averrhoa carambola $\mathrm{L}$. & Oxalidaceae & Kordoi & Cough & $\mathrm{T}$ & Fruit & $\begin{array}{l}\text { I: ripen fruit roasted in wood fire and eaten: juice } \\
\text { eaten directly }\end{array}$ \\
\hline 8. & Azadirachta indica A.Juss. & Meliaceae & Mohaneem & $\begin{array}{l}\text { Skin infection } \\
\text { /measles }\end{array}$ & $\mathrm{T}$ & Leaf & $\begin{array}{l}\text { E: mature leaves boiled with water and the water } \\
\text { used to bath. }\end{array}$ \\
\hline 9. & $\begin{array}{l}\text { Bacopa monnieri }(\mathrm{L} .) \\
\text { Wettst. }\end{array}$ & Scrophulariaceae & Brahmi & Brain tonic & $\mathrm{H}$ & $\begin{array}{l}\text { Whole } \\
\text { plant }\end{array}$ & I: whole plant grind and the juice drink. \\
\hline 10. & $\begin{array}{l}\text { Bryophyllum pinnatum } \\
\text { (Lam.) Oken }\end{array}$ & Crassulaceae & Duportenga & $\begin{array}{l}\text { Urinal } \\
\text { infection }\end{array}$ & $\mathrm{H}$ & Leaf & I: young leaf consumed directly. \\
\hline 11. & $\begin{array}{l}\text { Butea monosperma (Lamk.) } \\
\text { Taub }\end{array}$ & Fabaceae & Palas & Diarrhoea & $\mathrm{T}$ & Gum/resin & I: used directly \\
\hline 12. & $\begin{array}{l}\text { Calotropis gigantea }(\mathrm{L} .) \\
\text { Dryand. }\end{array}$ & Apocynaceae & Aah: Kam & Pain & $\mathrm{SH}$ & Leaf & $\begin{array}{l}\text { E: mature leaves kept over fire and then with } \\
\text { mastered oil and wrapped the pained area. }\end{array}$ \\
\hline 13. & Capsicum annuиm $\mathrm{L}$. & Solanaceae & Surging mirsi & $\begin{array}{l}\text { Stomach } \\
\text { problem/gastric }\end{array}$ & $\mathrm{SH}$ & Fruit & I: eaten directly \\
\hline 14. & Centella asiatica (L.) Urban. & Apiaceae & Manimuni & $\begin{array}{l}\text { Vomiting } \\
\text { /indigestion }\end{array}$ & $\mathrm{H}$ & $\begin{array}{l}\text { Whole } \\
\text { plant }\end{array}$ & $\begin{array}{l}\text { I: leaf juice with water or chewed } \\
\text { the whole plant. }\end{array}$ \\
\hline 15. & Cissus quadrangularis L. & vitaceae & Harjora & Bone fracture & CL & $\begin{array}{l}\text { Whole } \\
\text { plant }\end{array}$ & $\begin{array}{l}\text { E: the plant used as bandage or plastering for bone } \\
\text { fracture. }\end{array}$ \\
\hline 16. & $\begin{array}{l}\text { Citrus maxima (Burm.) } \\
\text { Merr. }\end{array}$ & Rutaceae & Singliang & Skin & $\mathrm{T}$ & Fruit & I: fruit eaten directly \\
\hline 17. & $\begin{array}{l}\text { Clerodendron colebrokianum } \\
\text { L. }\end{array}$ & Verbenaceae & Pakkom & $\begin{array}{l}\text { Weight } \\
\text { loss/fever }\end{array}$ & $\mathrm{SH}$ & Leaf & I: young leaf consumed as vegetable \\
\hline 18. & Corchorus capsularis L. & Tiliaceae & Mura & $\begin{array}{l}\text { Stomach probler } \\
\text { /vomiting }\end{array}$ & $\mathrm{SH}$ & Leaf & $\begin{array}{l}\text { I: tender Leaf dried with smoke and then boiled } \\
\text { and eaten }\end{array}$ \\
\hline
\end{tabular}




\begin{tabular}{|c|c|c|c|c|c|c|c|}
\hline $\begin{array}{ll}\text { SI. } \\
\text { No. }\end{array}$ & $\begin{array}{l}\text { Botanical name } \\
\text { of the plants }\end{array}$ & Family & $\begin{array}{l}\text { Vern name } \\
\text { (Mishing) }\end{array}$ & Uses & $\begin{array}{l}\text { Life } \\
\text { form }\end{array}$ & Part used & Dose/ administration $(\mathbf{E} / \mathbf{I})^{*}$ \\
\hline 19. & Datura stramonium $\mathrm{L}$. & Solanaceae & Dhatura & $\begin{array}{l}\text { Bite by mad } \\
\text { dog }\end{array}$ & $\mathrm{SH}$ & Root & I: root decoction \\
\hline 20. & Dillenia indica $\mathrm{L}$. & Dilleniaceae & Champa & Hair therapy & $\mathrm{T}$ & Fruit & $\begin{array}{l}\text { E: the seeds are grind and the then seeds are used } \\
\text { over hair for smooth and to reduce hair fall. }\end{array}$ \\
\hline 21. & $\begin{array}{l}\text { Drymaria cordata (L.) } \\
\text { Willd. ex Schult. }\end{array}$ & Caryophyllaceae & Laijabori & Dermatitis & $\mathrm{H}$ & leaf & I: leaf consumed directly. \\
\hline 22. & Eupatorium odoratum $\mathrm{L}$. & Asteraceae & Ayapan & $\begin{array}{l}\text { High blood } \\
\text { pressure }\end{array}$ & SH & Leaf/root & I: Decoction \\
\hline 23. & Ficus hirta Vahl & Moraceae & Taksek & Urine problem & $\mathrm{T}$ & Fruit & $\begin{array}{l}\text { I: ripen fruit used directly or cooked for urine } \\
\text { problem. }\end{array}$ \\
\hline 24. & Hedyotis diffusa Willd. & Rubiaceae & Sarpajiva & $\begin{array}{l}\text { Stomach pain/ } \\
\text { nerve tonic }\end{array}$ & $\mathrm{H}$ & Leaf & I: juice or cooked vegetable \\
\hline 25. & Jatropha curcas L. & Euphorbiaceae & Votera & Abortion & $\mathrm{T}$ & Resin & I: resin with milk consumed for $2 / 3$ days \\
\hline 26. & Justicia adhatoda L. & Acanthaceae & Bahaka & Cough & SH & Leaf & I: leaf juice used for dry cough \\
\hline 27. & Lawsonia inermis $\mathrm{L}$. & Lythraceae & Jetuka & $\begin{array}{l}\text { Skin and hair } \\
\text { diseases }\end{array}$ & $\mathrm{SH}$ & Leaf & E: leaf paste used in hair and skin \\
\hline 28. & Leucas aspera (Willd.) Link & Lamiaceae & Dorun & Sinus & $\mathrm{H}$ & Leaf & I: $2 / 3$ drop of leaf juice used per nose. \\
\hline 29. & Mangifera indica $\mathrm{L}$. & Anacardiaceae & Ke: di milong & Dysentery & $\mathrm{T}$ & Bark /seed & $\begin{array}{l}\text { I: decoction of bark taken in empty stomach; twice } \\
\text { daily before meal till complete relief. }\end{array}$ \\
\hline 30. & Melia azadirachta $\mathrm{L}$. & Meliaceae & Ghoraneem & Skin infection & $\mathrm{T}$ & Leaf & E: Leaf boiled and water used to bath. \\
\hline 31. & Moringa oleifera Lam. & Moringaceae & Munga & $\begin{array}{l}\text { Stomach } \\
\text { problem }\end{array}$ & $\mathrm{T}$ & $\begin{array}{l}\text { Fruit/flowe } \\
\text { r/leaf }\end{array}$ & I: cooked a vegetable \\
\hline 32. & $\begin{array}{l}\text { Murraya koenigii (L.) } \\
\text { Spreng. }\end{array}$ & Rutaceae & Norhing & Dysentery & $\mathrm{T}$ & Leaf & I: cooked a vegetable \\
\hline 33. & Nyctenthis arbor-tristis $\mathrm{L}$. & Oleaceae & Sewali & Worm & $\mathrm{T}$ & Flower & I: flower fry eaten \\
\hline 34. & Ocimum basilicum $\mathrm{L}$. & Lamiaceae & Tulsi & Cough & SH & Leaf & I: leaf juice with honey taken for cough. \\
\hline 35. & Paederia foetida $\mathrm{L}$. & Rubiaceae & Vedeli & Indigestion & $\mathrm{CL}$ & Leaf & I: leaf juice used directly or cooked with fish. \\
\hline 36. & $\begin{array}{l}\text { Phlogacanthus thyrsiflorus } \\
\text { Nees }\end{array}$ & Acanthaceae & Titaphul & Bronchitis & SH & $\begin{array}{l}\text { Bark \& } \\
\text { Leaf }\end{array}$ & $\begin{array}{l}\text { I: decoction of bark \& leaf taken till complete } \\
\text { relief. }\end{array}$ \\
\hline 37. & Phyllanthus acidus Skeel & Euphorbiaceae & Pora amlokhi & $\begin{array}{l}\text { White } \\
\text { discharge of } \\
\text { women }\end{array}$ & $\mathrm{T}$ & Leaf & $\begin{array}{l}\text { I: leaf juice with sugar. Daily in empty stomach for } \\
15 \text { days to } 3 \text { months. }\end{array}$ \\
\hline
\end{tabular}


Table 1. Details of the recorded ethno-medicinal plant species used by Mishing tribe. (Note: T- Tree, CL- Climber, SH- Shrub, H- Herb)

\begin{tabular}{|c|c|c|c|c|c|c|c|}
\hline $\begin{array}{l}\text { Sl. } \\
\text { No. }\end{array}$ & $\begin{array}{l}\text { Botanical name } \\
\text { of the plants }\end{array}$ & Family & $\begin{array}{l}\text { Vern name } \\
\text { (Mishing) }\end{array}$ & Uses & $\begin{array}{c}\text { Life } \\
\text { form }\end{array}$ & Part used & Dose/ administration \\
\hline 38. & Piper betel Blanco. & Piperaceae & Paan & Boil/cut injury & $\mathrm{CL}$ & stem & $\begin{array}{l}\text { E: stem is taken and dipped in the hot mustard oil } \\
\text { and then touched the boil or cut injury for relief of } \\
\text { pain and quick recovery }\end{array}$ \\
\hline 39. & Psidium guajava $\mathrm{L}$. & Myrtaceae & Madhuri & Stomach pain & $\mathrm{T}$ & Leaf & I: $2 / 3$ young leaf grind and $1 / 2$ spoon juice taken. \\
\hline 40. & Punica granatum $L$. & Lythraceae & Anar & $\begin{array}{l}\text { Diarrhoea/ } \\
\text { anaemia }\end{array}$ & SH & $\begin{array}{l}\text { Leaf/flowe } \\
\text { r/fruit }\end{array}$ & $\begin{array}{l}\text { I: fruit eaten directly; flower and leaf cooked; leaf } \\
\text { juice used }\end{array}$ \\
\hline 41. & Scoparia dulcis L. & Plantaginaceae & Tisilkosa & Diabetes & $\mathrm{SH}$ & Leaf & I: eaten directly. \\
\hline 42. & Sesamum orientalae L. & Padaliaceae & Tanam & Hair fall & SH & Seed & I: seed cooked or grind and used in hair. \\
\hline 43. & Streblus asper Lour. & Moraceae & Namhoi & Teeth problem & $\mathrm{T}$ & Stem & E: stem used as tooth brass. \\
\hline 44. & Swertia chirata Buds-Ham. & Gentinaceae & Sirata & $\begin{array}{l}\text { Worm /allergy } \\
\text { /vegetable / }\end{array}$ & $\mathrm{H}$ & Stem/ Leaf & $\begin{array}{l}2 / 3 \text { stem soaked for some hours or overnight and } \\
\text { the soaked water is taken ( } 2 \text { spoon) for two days }\end{array}$ \\
\hline 45. & Terminalia chebula Retz. & Combretaceae & Silika & $\begin{array}{l}\text { Hair growth } \\
\text { /constipation } \\
\text { /heart problem }\end{array}$ & $\mathrm{T}$ & Fruit & $\begin{array}{l}\text { I: Fruit dried and grind and the powder used } \\
\text { directly; or wholly eaten }\end{array}$ \\
\hline 46. & $\begin{array}{l}\text { Terminalia arjuna (Roxb. ex } \\
\text { DC.) Wight \& Arn. }\end{array}$ & Combretaceae & Arjun & $\begin{array}{l}\text { Heart/liver } \\
\text { tonic }\end{array}$ & $\mathrm{T}$ & Bark /root & $\begin{array}{l}\text { I: decoction of root and bark used; powder of bark } \\
\text { mixed with hot water for heart diseases. }\end{array}$ \\
\hline 47. & $\begin{array}{l}\text { Terminalia bellirica } \\
\text { (Gaertn.) Roxb. }\end{array}$ & Combretaceae & Bhomora & $\begin{array}{l}\text { Hair growth } \\
\text { /constipation }\end{array}$ & $\mathrm{T}$ & Fruit & $\begin{array}{l}\text { I: fruit dried and the inner part grind and the } \\
\text { powder used directly }\end{array}$ \\
\hline 48. & $\begin{array}{l}\text { Tylophora indica (Burm f.) } \\
\text { Merr. }\end{array}$ & Asclepiadaceae & Anantamul & $\begin{array}{l}\text { Liver Tonic/ } \\
\text { Jaundice }\end{array}$ & $\mathrm{CL}$ & Root & I: root eaten directly \\
\hline
\end{tabular}

Table 2. List of NTFPs (Dicots) marketed by Mishing tribe of Sonitpur, Assam. (Note: T- Tree, CL- Climber, SH- Shrub, H- Herb)

\begin{tabular}{|c|c|c|c|c|c|c|c|c|c|}
\hline $\begin{array}{l}\text { Sl. } \\
\text { No. }\end{array}$ & $\begin{array}{l}\text { Botanical name } \\
\text { of the plants }\end{array}$ & Family & $\begin{array}{l}\text { Vernacular name } \\
\text { (Mishing) }\end{array}$ & $\begin{array}{l}\text { Part } \\
\text { collected }\end{array}$ & Uses & $\begin{array}{c}\text { Life } \\
\text { forms }\end{array}$ & $\begin{array}{l}\text { Local market } \\
\text { price (INR) }\end{array}$ & $\begin{array}{l}\text { Source of } \\
\text { collection }\end{array}$ & Availability \\
\hline \multicolumn{10}{|c|}{ Fruits } \\
\hline 1. & $\begin{array}{l}\text { Baccaurea sapida (Roxb.) } \\
\text { Muell.-Arg. }\end{array}$ & Euphorbiaceae & Buri aaye & Fruit & Fruit & $\mathrm{SH}$ & $\begin{array}{l}15-20 \\
/ \text { dozen }\end{array}$ & Forest & March-July \\
\hline 2. & Citrus grandis (L.) Osbeck & Rutaceae & Sinkin & Fruit & Fruit & $\mathrm{T}$ & $5 /$ fruit & Forest & All seasons \\
\hline 3. & Garcinia paniculata Roxb. & Clusiaceae & Tepor tenga & Fruit & Fruit & $\mathrm{T}$ & 10/fruit & Forest & July-October \\
\hline 4. & $\begin{array}{l}\text { Phyllanthus acidus (L.) } \\
\text { Skeels }\end{array}$ & Phyllanthaceae & $\begin{array}{l}\text { Pomlokhi /Pora - } \\
\text { aamlokhi }\end{array}$ & Fruit & Fruit & $\mathrm{T}$ & $15-20 / \mathrm{kg}$ & Forest & March-May \\
\hline
\end{tabular}




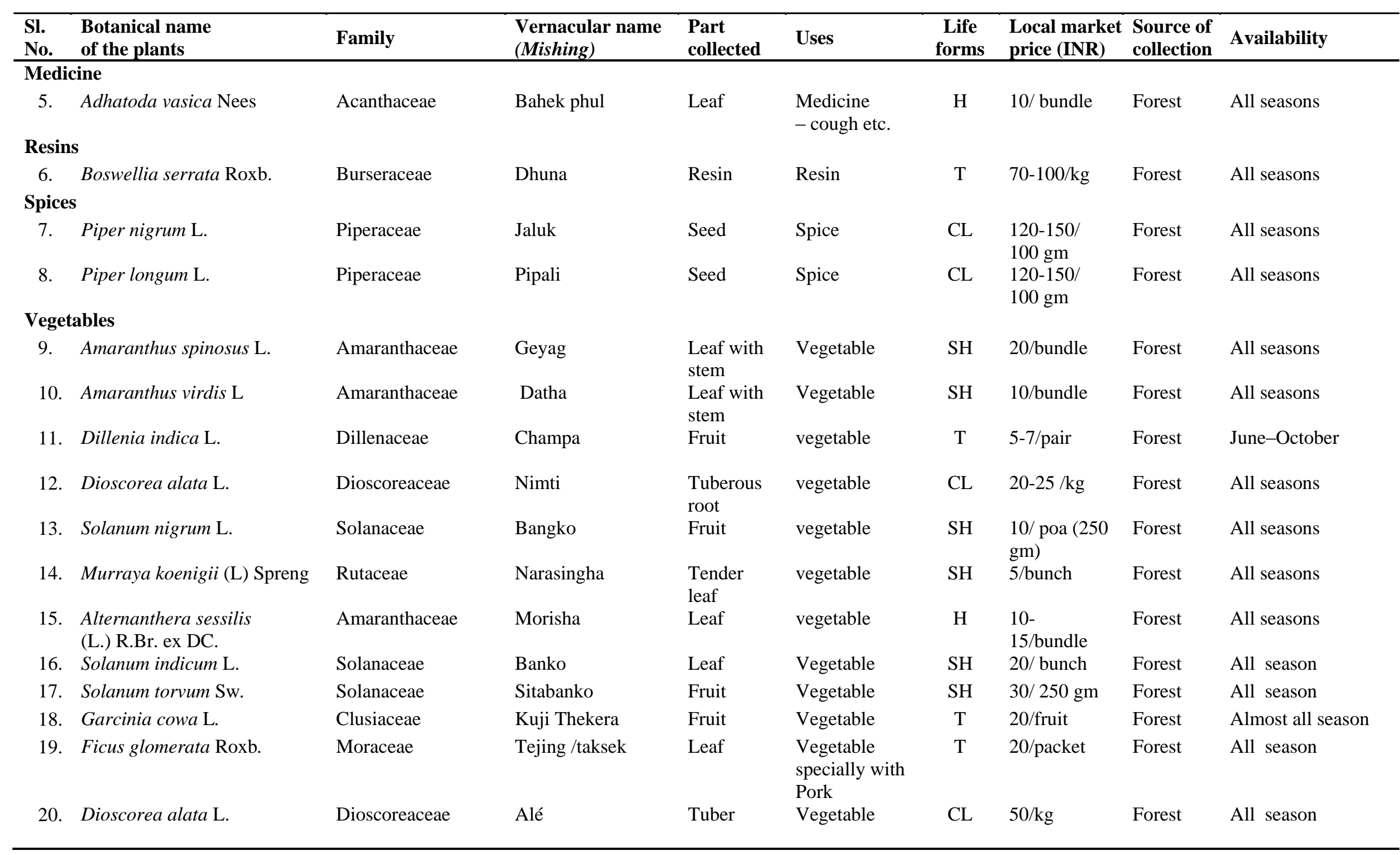




\begin{tabular}{|c|c|c|c|c|c|c|c|c|c|}
\hline $\begin{array}{l}\text { Sl. } \\
\text { No. }\end{array}$ & $\begin{array}{l}\text { Botanical name } \\
\text { of the plants }\end{array}$ & Family & $\begin{array}{l}\text { Vernacular name } \\
\text { (Mishing) }\end{array}$ & $\begin{array}{l}\text { Part } \\
\text { collected }\end{array}$ & Uses & $\begin{array}{c}\text { Life } \\
\text { forms }\end{array}$ & $\begin{array}{l}\text { Local market } \\
\text { price (INR) }\end{array}$ & $\begin{array}{l}\text { Source of } \\
\text { collection }\end{array}$ & Availability \\
\hline \multicolumn{10}{|c|}{ Vegetable And Medicine } \\
\hline 21. & Paederia foetida $\mathrm{L}$. & Rubiaceae & Bunka fore & $\begin{array}{l}\text { Young } \\
\text { Leaf }\end{array}$ & $\begin{array}{l}\text { Vegetable and } \\
\text { medicine for } \\
\text { stomach }\end{array}$ & $\mathrm{CL}$ & 10/bundle & Forest & All seasons \\
\hline 22. & Centella asiatica (L.) Urban & Apiceae & Manimuni & $\begin{array}{l}\text { Whole } \\
\text { plant }\end{array}$ & $\begin{array}{l}\text { problem. } \\
\text { medicine and } \\
\text { vegetable }\end{array}$ & $\mathrm{H}$ & 5/bundle & Forest & All seasons \\
\hline 23. & Leucas aspera (Willd) Link & Lamiaceae & Durum & $\begin{array}{l}\text { Young } \\
\text { leaf }\end{array}$ & $\begin{array}{l}\text { vegetable and } \\
\text { medicine for } \\
\text { nose problem }\end{array}$ & $\mathrm{H}$ & 5/bunch & Forest & All seasons \\
\hline
\end{tabular}

Table 3. List of plant species (dicots) used for the preparation of Epop, used for preparation of Nogjin Apong. (Note: T- Tree, CL- Climber, SH- Shrub, H- Herb)

\begin{tabular}{|c|c|c|c|c|c|c|}
\hline Sl. No. & $\begin{array}{l}\text { Botanical name } \\
\text { of the plants }\end{array}$ & Family & $\begin{array}{l}\text { Vern name } \\
\text { (Mishing) }\end{array}$ & Life form & Part used & Use /mode \\
\hline 1. & Clerodendrum infortunatum $\mathrm{L}$. & Verbenaceae & Pakkom & $\mathrm{SH}$ & Leaf & $\begin{array}{l}\text { I: Leaf dried and powder used in the } \\
\text { preparation of Epop }\end{array}$ \\
\hline 2. & Coriandrum sativum $\mathrm{L}$. & Apiaceae & Dhania & $\mathrm{H}$ & Stem/leaf & $\begin{array}{l}\text { I: stem/ Leaf dried and powder used } \\
\text { in the preparation of Epop }\end{array}$ \\
\hline 3. & Costus speciosus (J.Koenig) Sm. & Costaceae & Jomlakhuti & $\mathrm{SH}$ & Leaf & \\
\hline 4. & Cuscuta reflexa Roxb. & Convolvulaceae & Rabonlota & $\mathrm{CL}$ & Root & I: root dried and powder used in the \\
\hline 5. & Flemingia strobilifera (L.) W.T.Aiton & Fabaceae & Makhioti & $\mathrm{SH}$ & Leaf & preparation of Epop \\
\hline 6. & Hibiscus rosa-sinensis L. & Malvaceae & Leunaapum & $\mathrm{T}$ & Leaf & $\begin{array}{l}\text { I: Leaf dried and powder used in the } \\
\text { preparation of Epop }\end{array}$ \\
\hline 7. & Lippia javanica (Burm.f.) Spreng. & Verbenaceae & ----- & $\mathrm{H}$ & Leaf/flower & $\begin{array}{l}\text { I: Leaf/flower dried and powder } \\
\text { used in the preparation of Ерор }\end{array}$ \\
\hline 8. & Polygonum hydropiper L. & Polygonaceae & Leubo & $\mathrm{H}$ & Leaf/stem & $\begin{array}{l}\text { I: Leaf/stem dried and powder used } \\
\text { in the preparation of Еpop }\end{array}$ \\
\hline 9. & Polygonum microcephalum D. Don & Polygonaceae & Nekungkune & $\mathrm{H}$ & Leaf & ---- \\
\hline 10. & Tinospora cordifolia (Willd.) Miers & Menispermaceae & Amrita & $\mathrm{CL}$ & Leaf & I: Leaf dried and powder used in the \\
\hline 11. & Zanthoxylum nitidum (Roxb.) DC. & Rutaceae & Jabrang & $\mathrm{SH}$ & Leaf & preparation of Epop \\
\hline
\end{tabular}


recorded as NTFPs that has been marketed extensively by this community in different regions of the district (Table 2). It has been observed that species used for vegetables contributes maximum (52\%) followed by fruits (18\%); species used for both vegetable and medicine (13\%); spices (9\%); and resins and medicine 4\% each (Fig. 5). Different plant parts were collected by the villagers for marketing and to be used for vegetables, fruits, resins, medicine, etc. The majority of the plant parts are available throughout the year and some are found to be seasonal. One more interesting fact about the marketing of the NTFPs is that they generally do not use the weighing scale for measuring quantity of items instead they sold the items in some bundles/bunch or in pairs.

Another most important traditional practice of Mishing community is the preparation of Nogin or Nogjin Apong, a traditional rice alcoholic beverage of Mishing Community. For the preparations of the Nogin Apong they use many plants species (both dicots and monocots). During the preparations of the Apong first they prepare Epop. Eрор is the tablet or ball shaped cake prepared with the dry powder of plant materials mixing with the rice powder and is used for the preparation of Nogin or Nogjin Apong. During the study a total of 11 dicot plant species (Clerodendrum infortunatum, Coriandrum sativum, Costus speciosus, Cuscuta reflexa, Flemingia strobilifera, Hibiscus rosa-sinensis, Lippia javanica, Polygonum hydropiper, P. microcephalum, Tinospora cordifolia and Zanthoxylum nitidum) under 10 genera and 9 families (Table 3) were recorded that were exclusively used for the preparation of Epop.

\section{DISCUSSIONS}

The main objective of the present study was to investigate the different plant species (especially dicots) that are exclusively used by the Mishing community in their day-to-day life for different purposes like medicine, vegetable and importantly other NTFPs i.e. vegetables, fruits, medicine, spices, gum/resin etc. as their one of the important income sources. From the result it has been observed that the majority of the plant species were used mainly for medicinal purpose. Leaves exhibited major part of the plant used for treatment of majority of diseases contributing 50\% while flower shows lowest used (2\%). Saikia et al. (2010) studied ethnobotany of Bodo tribes in Sonitpur district and enumerated 20 species and analysed briefly the degree of dependency on medicinal plants and common health concern. Another etnobotanical study reported a total number of 20 plant species out of which 12 dicot species from Gahpur area of Sonitpur district (Saikia 2006). In the present study a total of 48 plant species were recorded for medicinal uses, 23 species were recorded as NTFPs and 11 species recorded that are used for the preparation of Apong. Majority of the plants like Ananas comosus, Centella asiatica, Psidium guajava, Hedyotis diffusa, Moringa oleifera, Corchorus capsularis, Capsicum annuит, Streblus asper and Asparagus racemosus are used for stomach problems while Butea monosperma, Punica granatum, Acacia nilotica, Mangifera indica, Murraya koenigii, etc are used for dysentery. Averrhoa carambola, Justicia adhatoda, Ocimum basilicum and Adhatoda vasica are commonly used for cold and cough by the community. Fruits of Baccaurea sapida, Citrus grandis, Garcinia paniculata, Phyllanthus acidus, etc are marketed as NTFPs while resins of Boswellia serrate and fruit/seeds of Piper nigrum and P. longum are marked as spices.

\section{CONCLUSION}

From the present study it can be concluded that the Mishing tribe is highly dependent upon the dicot angiosperms as their source of income in addition to fulfilling their various day to day requirements. Thus NTFPs plays a major role in the livelihood status of Mishing tribe by supplying domestic requirement and marketing goods. Understanding the present days environmental health issues related to chemicals in the form of pesticides, weedicide, etc. peoples generally prefer to buy the NTFPs sold in the local markets by tribal peoples which are collected from the forest. This marginal marketing also serves as a source of income to this community.

\section{ACKNOWLEDGEMENTS}

Authors are thankful to the villagers of Dharikati, Khonamukh, Kathani, Rangajan, Rongajan miri, Baligaon, Sotaimiri, Toupamiri, Bamunipam, Bordikorai, Sikomgaon, Silenighat, Morikhuti, Bokagaon, Kekokoli, Tinighoria and Gudamghat villages of Sonitpur district of Assam, India. Author offers their sincere thanks to Ms. N Pamegam (ATIO, Tezpur), Mr. M Das (ATIO, Bhalukpong) for their tremendous help throughout the field work. Authors deeply acknowledged the help of Comission Mili, Podeswar Mili, Noipani Doley, Kameswar Mili, Dimbeswar Mili, Bismita Doley and Joinath Gum for their assistance during survey. 
Special thanks to Dr. GC Sarma, Dept. of Botany, G.U. for identification of plant species and Dr. Rajesh Kumar Sah of GIS Lab., Dept. of Environmental Science, Tezpur University for their kind help.

\section{REFERENCES}

Bajpai O, Pandey J \& Chaudhary LB (2016) Ethnomedicinal Uses of Tree Species by Tharu Tribes in the Himalayan Terai Region of India. Research Journal of Medicinal Plant 10 (1): 19-41.

Census of India (2011) Office of the Registrar General \& Census Commissioner, India.

Chopra K (1993) The Value of Non-Timber Forest Products: An Estimation for Tropical Deciduous Forests in India. Economic Botany 47: 251-257.

Das AK (2008) Medicinal plants used by different tribes of Cachar district, Assam. Indian Journal of Traditional Knowledge 7 (3): 446-454.

Datta T, Patra AK \& Dastidar SG (2014) Medicinal plants used by tribal population of Coochbehar district, West Bengal, India-an ethnobotanical survey. Asian Pacific Journal Trop Biomed 4 (1): 478-482.

Dutta BK \& Dutta PK (2005) Potential of ethnobotanical studies in North East India: an overview. Indian Journal of Traditional Knowledge 4 (1): 7-14.

Fransworth NR (1988) Screening plants for new medicines. In: Wilson E O (ed) Biodiversity. National Academy Press, Washington DC, USA, pp. 212-216.

Gauraha AK (1992) Micro-Economic Analysis of a Tribal Village. Indian Journal of Agricultural Economics 47(3): 446-447.

Hooker JD (1875-1894) Flora of British India, Vols. 1-6. L. Revee \& Co., London.

Jain DL, Baheti AM, Jain SR \& Khandelwal KR (2010) Use of medicinal plants among tribes in Satpuda region of Dhule and Jalgaon districts of Maharashtra-an ethnobotanical survey. Indian Journal of Traditional Knowledge 9 (1): $152-157$.

Jain SK \& Borthakur SK (1980) Ethnobotany of the Mikirs of India. Economic Botany 34: 264.

Jain SK \& Rao RR (1977) A handbook of field and herbarium methods. Today and Tomorrow's printers and publishers, Delhi, India, $196 \mathrm{p}$.

Jain SK (1987) A manual of Ethnobotany. Scientific publisher, Jodhpur, India, 242 p.

Jain SK (1989) Methods and approaches in Ethnobotany. Society of Ethnobotanists, Lucknow, India, 192 p.

Jeyaprakash K, Ayyanar M, Geetha KN \& Sekar T (2011) Traditional uses of medicinal plants among the tribal people in Theni districts (Western Ghats), Southern India. Asian Pacific Journal of Tropical Biomedicine 1(1): $20-25$.

Kala CP (2005) Current status of medicinal plants used by traditional vaidyas in Uttaranchal State of India. Ethnobotanical Research Applications 3: 267-278.

Kanjilal UN, Kanjilal PC, Das A \& De RN (1934-1940) Flora of Assam, Vols. 1-4, Govt. Press, Shillong.

Mallik RH (2000) Sustainable Management of Non-Timber Forest Products in Orissa: Some Issues and Options. Indian Journal of Agricultural Economics 55 (3): 384-397.

Mehra A, Bajpai O \& Joshi H (2014) Diversity, utilization and sacred values of Ethno-medicinal plants of Kumaun Himalaya. Tropical Plant Research 1(3): 80-86.

Ngbolua KN, Mihigo SO, Liyongo CI, Ashande MC, Tshibangu DST, Zoawe BG, Baholy R, Fatiany PR \& Mpiana PT (2016) Ethno-botanical survey of plant species used in traditional medicine in Kinshasa city (Democratic Republic of the Congo). Tropical Plant Research 3(2): 413-427.

Rao GN (1987) Significance of Minor Forest Produce in Tribal Economy: A Case Study. Kurukshetra 7: 23-28.

Rout J (2012) Medicinal plants of North cachar hill district of Assam used by Dimasa tribe. Indian Journal of Traditional Knowledge 11 (3): 520-527.

Saikia B (2006) Ethnomedicinal plants from Gahpur of Sonitpur district. Indian Journal of Traditional Knowledge 5(4): 529-530.

Saikia B, Borthakur SK \& Saikia N (2010) Medico-ethnobotany in Bodo tribes in Gahpur of Sonitpur district, Assam. Indian Journal of Traditional Knowledge 9(1): 52-54.

Shiva MP (1993) Solutions to overcome impediments in forest development through minor forest product based management. In: International Seminar on MFP in Forestry, Held on 17-18 April 1993, Dehra Dun, India.

Singh U \& Lahiri N (2010) Ancient India: new research. Oxford University Press, New Delhi, India, 320p.

Teron R \& Borthakur SK (2014) Ethnobotanical appraisal of the Hill-Tiwas of Assam, India. Pleione 8(1): 109-119.

WHO (2002-2005) World Health Organization traditional medicine strategy Geneva: World Health Organization. Available from: tp://www.who.int/medicines/ publications/ traditional policy/en/index.htm. (accessed: 12 Jun. 2016). 